\title{
罗非昔布及其衍生物合成研究进展
}

\author{
刘改枝*, 韩德恩 ${ }^{a}$ 王 鹏 $b$ 刘宏民 ${ }^{*}, b$ \\ $\left({ }^{a}\right.$ 河南中医学院药学院 郑州 450046) \\ ( ${ }^{b}$ 郑州大学药学院 郑州 450001)
}

\begin{abstract}
摘要 罗非昔布是一种新型非甾体抗炎药，是环氧化酶抑制剂的代表性药物，服用罗非昔布会增加患心脏病或中风的 危险. 虽然罗非昔布曾由于心血管安全风险而撤市，但其优异的镇痛抗炎活性仍然具有很大的研究价值和广阔的应用 前景, 因此以罗非昔布为先导寻找具有更高药理活性或更低毒副作用的罗非昔布衍生物一度成为研究热点. 综述了罗 非昔布及其衍生物的研究进展，包括罗非昔布合成工艺的研究，罗非昔布衍生物的合成.
\end{abstract}

关键词 罗非昔布; 衍生物; 合成; 研究进展

\section{Synthesis Research Progress of Rofecoxib and Its Derivatives}

\author{
Liu, Gaizhi ${ }^{*, a}$ Han, Deen ${ }^{a} \quad$ Wang, Peng ${ }^{b} \quad$ Liu, Hongmin ${ }^{*, b}$ \\ $\left({ }^{a}\right.$ Henan University of TCM, Zhengzhou 450046) \\ ( $b$ School of Pharmaceutical Science, Zhengzhou University, Zhengzhou 450001)
}

\begin{abstract}
Rofecoxib is a novel non-steroidal anti-inflammatory drug, which is the representative of cyclooxygenase inhibitor. Taking rofecoxib would increase the risk of heart attack or stroke risk. Although rofecoxib withdrawal from the market due to cardiovascular risk, but its excellent analgesic and anti-inflammatory activity still has great research value and broad application prospect. Therefore, rofecoxib as a lead compound, has become a focus of research, from which researchers try to find good activity and little side effects rofecoxib derivatives. The research progress of rofecoxib and its derivatives is reviewed, including synthesis of rofecoxib and its derivatives.
\end{abstract}

Keywords rofecoxib; derivative; synthesis; research progress

罗非昔布(rofecoxib)，化学名为 4-[4-(甲磺酰基)苯 基]-3-苯基-2-(5H)-呋喃酮, 代号 MK-0966, 商品名为 Vioxx, 由美国 Merck 公司开发, 于 1999 年在墨西哥首 先上市. 本品选择性抑制 COX-2, 用于治疗骨关节炎 ${ }^{[1]}$, 控制成人锐痛和痛经 ${ }^{[2,3]}$, 但胃肠道不良反应如溃病、出 血和穿孔等可显著减少 ${ }^{[4 \sim 7]} .2003$ 年全球销售额达 25 亿 美元. 但由于服用罗非昔布会增加患心脏病或中风的危 险, 2004 年 9 月 30 日默沙东公司美国总部(默克公司)宣 布了在全球范围内主动撤回 Vioxx ${ }^{[8]} .2005$ 年 2 月 18 日, 美国食品药品监督管理局(FDA)专家顾问委员会经过会 议研讨，通过投票方式同意已撤出市场的 rofecoxib 再 度上市 ${ }^{[9]}$. 由于其良好的抗炎活性及安全性, 对其进行 结构改造试图寻找具有相似生物活性且副作用小的衍 生物一度成为人们研究的热点, 国内外研究人员通过对
其构效关系研究，设计合成了多种类型的类似物及衍生 物, 本文对罗非昔布及其衍生物的合成进行概述, 并且 对其主要衍生物的合成及生物活性作简要评述.

\section{1 罗非昔布合成工艺研究进展}

Desmond 等 ${ }^{[10]}$ 于 1996 年报道了以苯乙酸(2)为起始 原料经过五步反应得到目标化合物罗非昔布(Scheme 1 所示). 首先苯乙酸 (2)与溴代乙酸乙酯经缩合、环合得到 3-苯基-4-差基呋喃酮(4), 化合物 $\mathbf{4}$ 的羟基用三氟甲磺酸 酐保护得化合物 $\mathbf{5}$, 化合物 $\mathbf{5}$ 再与 4-甲硫基苯基硼酸缩 合得 4-(4-甲硫基)苯基-3-苯基-2-(5H)-呋喃酮(6), 化合 物 6 经臭氧氧化得目标化合物罗非昔布 1. 本路线的总 收率为 $45 \%$.

Atkinson 等 ${ }^{[11]}$ 于 1996 年报道了以呋喃酮 7 与对甲

*E-mail: liugaizhi@126.com; liuhm@zzu.edu.cn

Received December 30, 2012; revised February 5, 2013; published online February 22, 2013.

Project supported by the Doctoral Research Foundation of Henan University of Traditional Chinese Medicine (No. BSJJ2010-03).

河南中医学院博士基金(No. BSJJ2010-03)资助项目. 


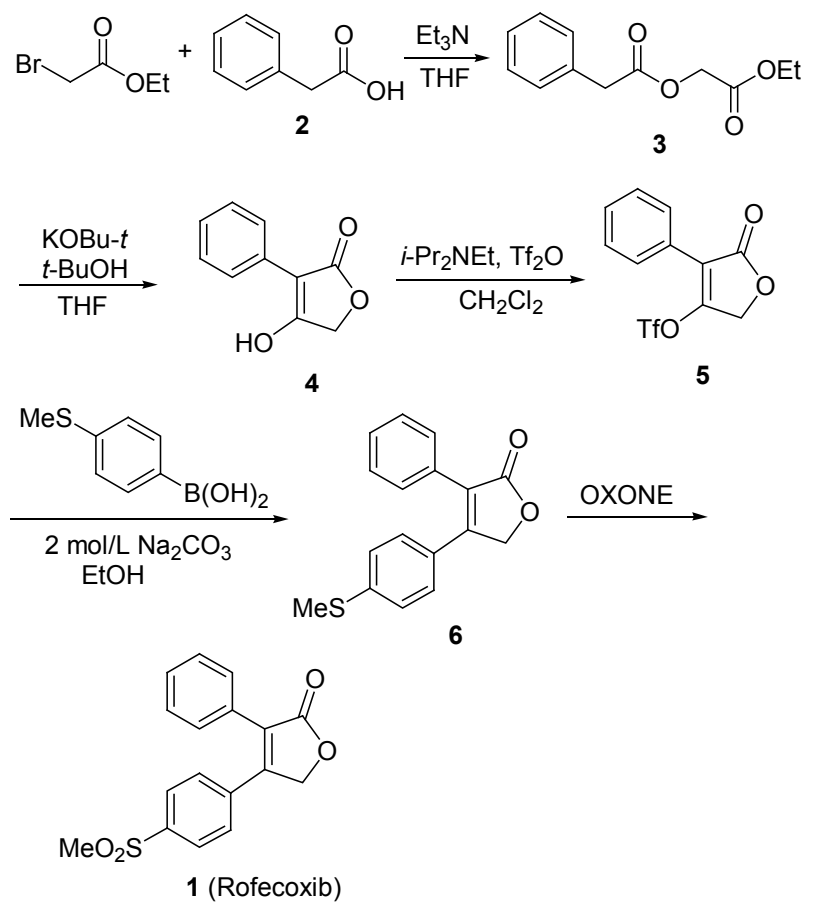

Scheme 1

硫基苯的格氏试剂 8 为起始原料经五步反应得到目标化 合物罗非昔布(Scheme 2 所示). 首先用呋喃酮 7 与对甲 硫基苯的格氏试剂 8 在卤化铜与三甲基氯硅烷条件下反 应得到化合物 9 , 化合物 9 氧化得到化合物 $\mathbf{1 0}$, 化合物 10 碘代得到化合物 11, 化合物 11 与苯基嗍酸反应得到 4-(4-甲硫基)苯基-3-苯基-2-(5H)-呋喃酥(6), 6 在臭氧条 件下氧化得到目标化合物罗非昔布 1 . 本路线的总收率 为 $45 \%$.

Desmond 等 ${ }^{[12]}$ 于 1998 年报道了以苯甲硫醚为起始 原料经过五步反应得到目标化合物罗非昔布(Scheme 3 所示). 首先苯甲硫醚(12)在无水三氯化铝及邻二氯苯存 在条件下与乙酰氯发生傅-克酰基化反应生成对甲硫基 苯乙酮(13), 对甲硫基苯乙酮(13)在铇酸钠及过氧化氢 存在下发生氧化反应生成对甲磺酰基苯乙酮(14), 然后 在 48\%氢溴酸引发下用液溴溴代对甲磺酰基苯乙酮(14) 得到 $\alpha$-溴代-对甲磺酰基苯乙酮(15), 最后在碱性条件 下与苯乙酸缩合一锅法得到目标化合物罗非昔布(1). 本路线的总收率为 $58 \%$.

Thérien 等 ${ }^{[13]}$ 于 2001 年报道了同样以苯甲硫醚为起 始原料经过五步反应得到目标化合物罗非昔布(Scheme 4 所示). 第一步与路线三相同, 苯甲硫醚 (12)与乙酰氯 在无水三氯化铝及邻二氯苯存在条件下发生傅-克酰基 化反应生成对甲硫基苯乙酩(13), 对甲硫基苯乙酮(13) 用过氧化钛酸镁(MMPP)氧化得对甲磺酰基苯乙酩(14), 然后在三氯化铝催化下用液溴溴代对甲磺酰基苯乙酮 (14)得到 $\alpha$-溴代-对甲磺酰基苯乙酮(15), 化合物 15 在

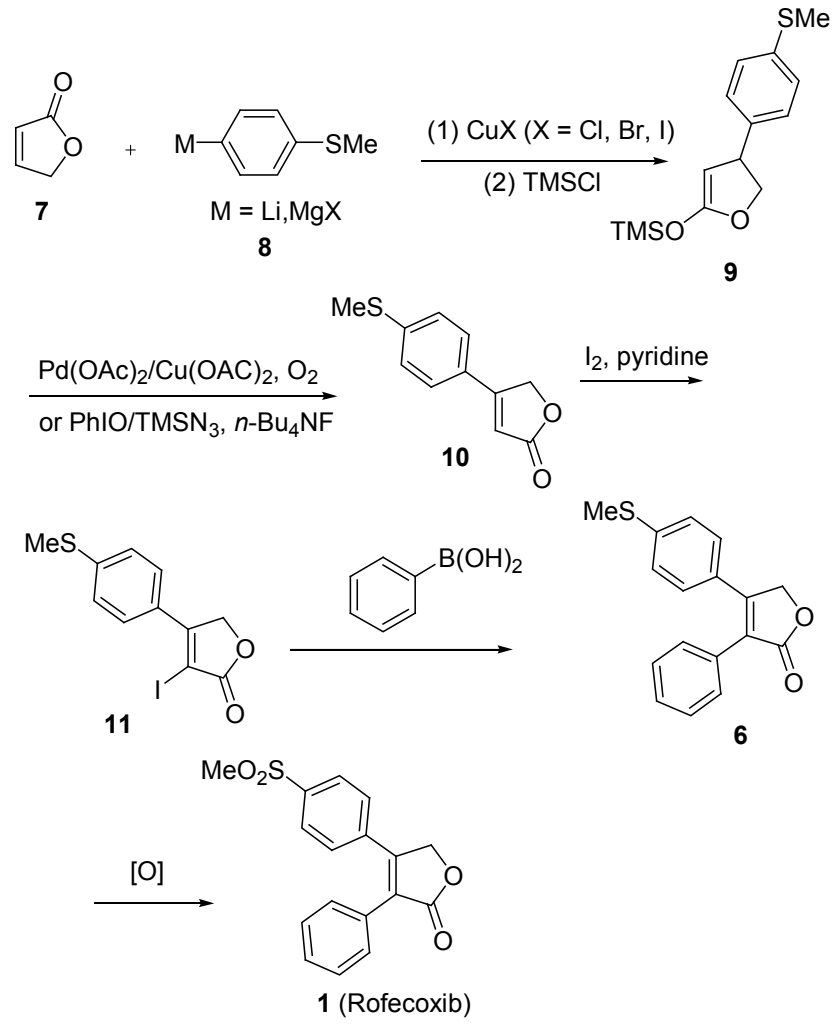

Scheme 2<smiles>CC(=O)c1ccccc1Cl</smiles><smiles>CO[R16](=O)c1ccc(C2=C(c3ccccc3)C(=O)OC2)cc1</smiles>

Scheme 3

弱碱性条件下与苯乙酸缩合得到化合物 16 , 最后在 DBU 存在下环合得到目标化合物罗非昔布(1). 本路线 总收率为 $59 \%$.

敖桂珍等 ${ }^{[14]}$ 对罗非昔布合成工艺进一步改进，于 2002 年报道了与路线三相类似的合成路线. 同样以苯 甲硫醚(12)为起始原料经过五步反应得到目标化合物罗 非昔布(Scheme 5). 在第一步中选用了氯仿代替邻二氯 苯做溶剂进行傅-克酰化反应，同时第二步选用浓硫酸 


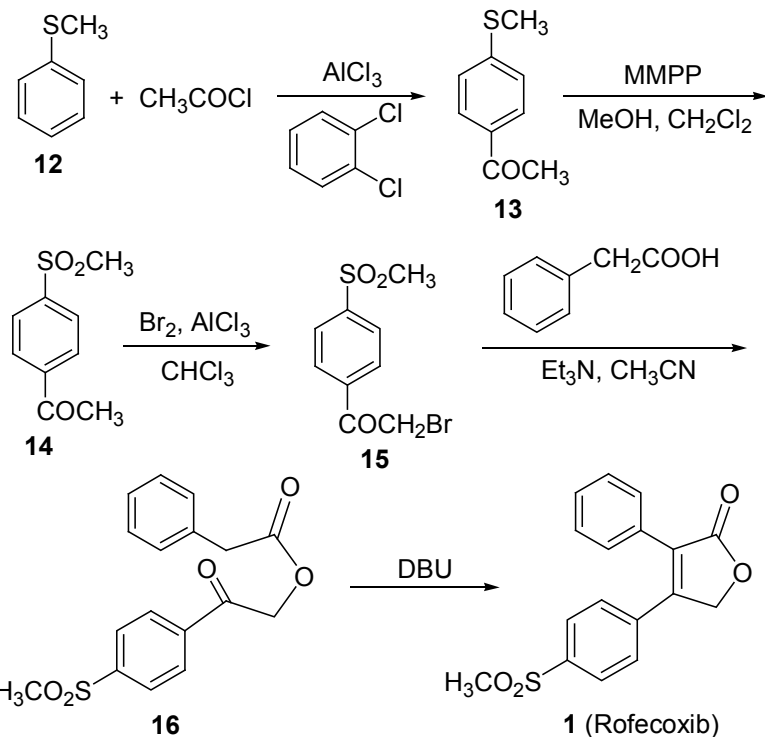

Scheme 4

作为氧化反应的催化剂, 后三步反应均与路线三相同, 五步总收率为 $58 \%$.

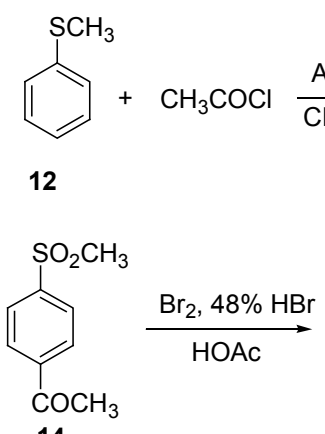

14<smiles>COS(=O)(=O)c1ccc(C(=O)COC(=O)Cc2ccccc2)cc1</smiles>

$\underset{\mathrm{CH}_{2} \mathrm{Cl}_{2}}{\stackrel{\mathrm{AlCl}_{3}}{\longrightarrow}}$<smiles>Cc1ccc(C(C)(C)C)cc1</smiles>

13<smiles>COS(=O)(=O)c1ccc(C(=O)O)cc1</smiles>

15

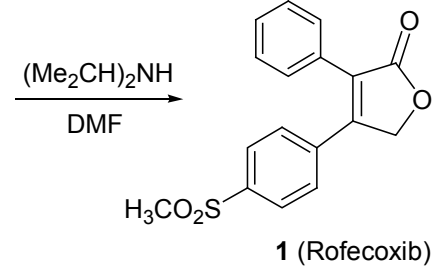

Scheme 5

在上述合成路线中, 路线一在环合一步用到叔丁醇 钾, 易受潮变质, 反应条件要求苛刻, 同时使用价格昂 贵的 4-甲硫基苯基嗍酸成本较高. 路线二需要使用多种 昂贵的试剂，如卤代铜、醋酸铅、单质碘、苯基硼酸等， 成本较高, 同时起始原料呋喃酮及对甲硫基苯的格氏试 剂均较难得到. 路线三需要使用价格昂贵的铇酸钠, 同 时邻二氯苯对环境污染较大. 路线四用到 $\operatorname{MMPP}($ 过氧 化钛酸镁)进行氧化, 同时使用价格昂贵的 DBU 作为缩 合剂, 成本较高. 路线五原料价廉易得, 各步所用试剂 均较便宜, 反应条件相对温和, 操作简单, 适合工业化 生产.

\section{2 罗非昔布衍生物的合成研究进展}

\section{1 丁烯内酯环修饰的衍生物}

二芳基杂环类是 COX-2 选择性抑制剂中研究最多 的一类(图 1), Gans 等 ${ }^{[15]}$ 研制的 DUP-697 是其原型药物. $\mathrm{COX}$ 选择性测试证实本品对 COX-2 具有较高选择性 $\left(\mathrm{COX}-2: K_{i}=0.3 \mu \mathrm{mol} / \mathrm{L}, \mathrm{COX}-1 ; K_{i}=5.3 \mu \mathrm{mol} / \mathrm{L}\right)^{[16]}$. 动 物试验结果显示, 化合物 DuP-697 为强效抗炎剂, 其抑 制大鼠佐剂关节炎和足趾肿胀的 $\mathrm{ED}_{50}$ 分别为 0.18 和 $0.03 \mathrm{mg} \cdot \mathrm{kg}^{-1}$, 本品具有较好胃肠道耐受性 ${ }^{[15]}$.<smiles>Bc1cc(-c2ccc(S(=O)(=O)OC)cc2)c(-c2ccc(F)cc2)s1</smiles>

DuP-697<smiles>NS(=O)(=O)c1ccc(-n2nc(C(F)(F)F)cc2-c2ccc(Cl)cc2)cc1</smiles><smiles>COSc1ccc(-n2nc(C(F)(F)F)cc2-c2ccc(F)cc2)cc1</smiles>

图 1 代表性二芳基杂环类 COX-2 选择性抑制剂

Figure 1 Representative double aromatic heterocyclic COX-2 selective inhibitors

SC-58635 (YM-177, Celecoxib) 是第一个上市的 COX-2 选择性抑制剂: SC-236 是 Searle 公司早期合成的 一个 1,5-二芳基吡唑类 COX-2 抑制剂，但由于其半衰期 过长, 毒性太大而被淘汰. 为了改善 SC-236 药动学方面 的不足, Searle 公司对 1,5-二芳基吡唑类化合物进行了 广泛的构效关系考察, SC-58635 就是在这种情况下开发 出来的一个高效 COX-2 选择性抑制剂, 其对 COX-2 的 IC50 仅为对 COX-1 的 $1 / 400^{[17]}$. 主要用于关节炎 $(\mathrm{OA})$ 和类风湿性关节炎(RA). 药动学研究发现本品起效时间 短，与传统 NSAIDs 比较，其溃疡发生率与肾脏毒性都 显著降低.

作为 Searle 公司开发 COX-2 选择性抑制剂的一个 “模板”化合物, SC-58125 对多种慢性炎症均呈现出强的 口服活性，如抑制大鼠佐剂关节炎的 $\mathrm{ED}_{50}$ 为 $0.4 \mathrm{mg}$ • $\mathrm{kg}^{-1}$, 而且胃肠道毒副作用小于传统 NSAIDs ${ }^{[18]}$. 体外 选择性测试结果显示, SC-58125 抑制白介素-1 (IL-1)诱 导的成纤维细胞中 COX-2 和血小板中 $\mathrm{COX}-1$ 的 $\mathrm{IC}_{50}$ 分 别为 0.07 和 $100 \mu \mathrm{mol} / \mathrm{L}, \mathrm{COX}-2 / \mathrm{COX}-1$ 选择系数大于 1400 倍 ${ }^{[19]}$, 为一个 COX-2 高选择性抑制剂.

以不同杂环取代的衍生物，如 Rofecoxib ${ }^{[20] ， E t o r i-~}$ 
coxib, Valdecoxib ${ }^{[21]}$ 和 parecoxibs odium(图 2)均已上市. 其中 Etoricoxib 和 Valdecoxib 为第二代 COX-2 抑制剂, 特异性更高, 副作用更小. Parecoxibs odium 是 valdecoxib 的酰胺化前体, 为注射用选择性 COX-2 抑制剂, 用于手术或创伤的疼痛, 为非胃肠道使用的中至重度镇 痛剂 ${ }^{[22 \sim 24]}$.

文献[25]报道合成了系列具有潜在 COX-2 抑制活 性的新型 4-(4-甲磺酰基苯基)-3-苯基-2(3H)-噻唑硫酮衍 生物, 合成路线如 Scheme 6 所示, 通式 I 即为新型二芳 基杂环衍生物.

Engelhardt 等 ${ }^{[26]} 2006$ 年合成了一种开环的罗非昔布
衍生物 1, 这种开环的衍生物作为罗非昔布的前药可以 在体内释放原药和 NO, 后者对胃肠道具有保护作用, 可以减少或消除罗非昔布引起的胃肠道副作用(Scheme 7).

这种前药以 3-苯基-2-丙炔基-1-醇为原料, 经过 5 步 反应合成目标化合物，总收率为 $64 \%$ ，合成路线如 Scheme 8 所示.

另外罗非昔布的开环衍生物有如下图通式及图 3 所示的研究报道. 化合物 $\mathbf{I I}_{\mathbf{1}} \sim \mathbf{I I}_{\mathbf{1 7}}$ 抗炎活性结果表明, 罗非昔布开环衍生物大部分具有较强的抗炎活性, 而且 胃肠道副作用显著小于双氯芬酸, 但略大于罗非昔布.

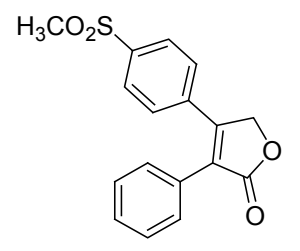

Rofecoxib (1)<smiles>COS(=O)(=O)c1ccc(-c2cc(Cl)cnc2-c2ccc(C)nc2)cc1</smiles>

Etoricoxib<smiles>Cc1onc(-c2ccccc2)c1-c1ccc(S(N)(=O)=O)cc1</smiles>

Valdecoxib<smiles>CCOCOc1ccc(-c2c(-c3ccccc3)noc2C)cc1</smiles>

Parecoxib sodium

图 2 代表性 COX-2 选择性抑制剂

Figure 2 The representative COX-2 selective inhibitors

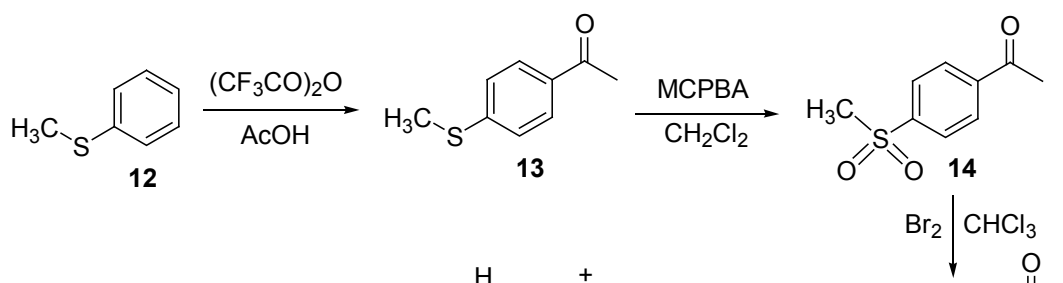

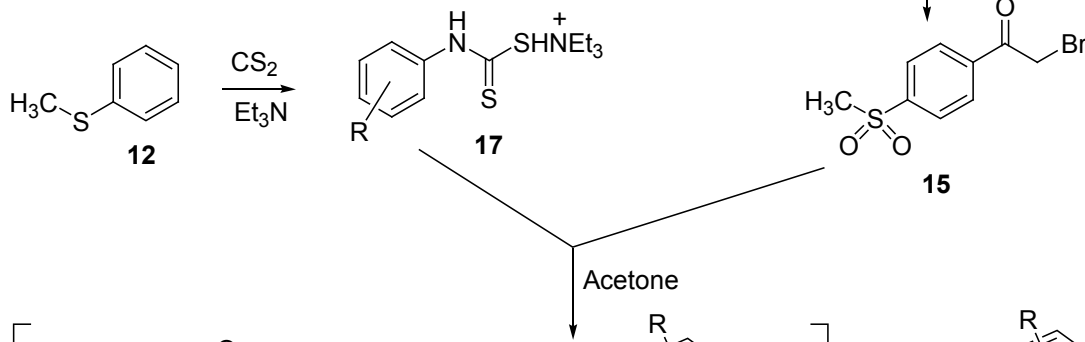

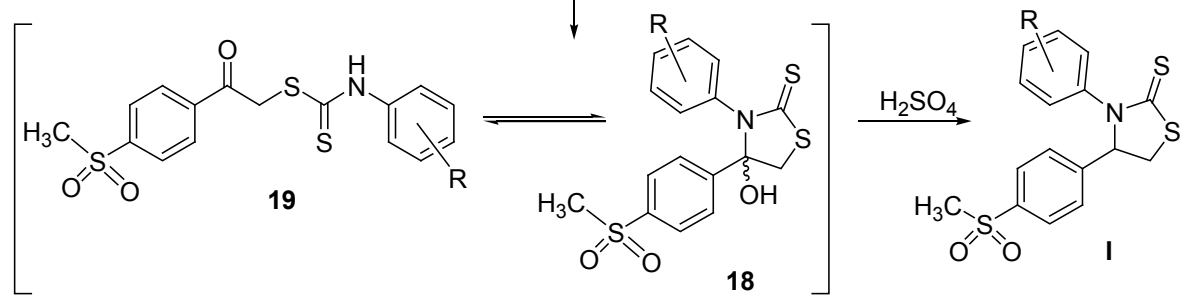

a: $R=H ; \mathbf{b}: R=4-B r ; \mathbf{c}: R=3-B r ; \mathbf{d}: R=4-C l ; \mathbf{e}: R=3,4-C_{2} ; \mathbf{f}: R=4-F ; \mathbf{g}: R=3-F ; \mathbf{h}: R=4-M e$

Scheme 6

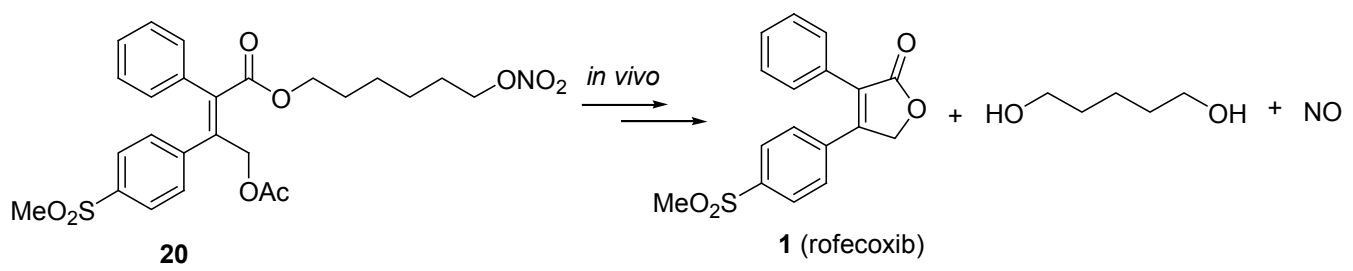

Scheme 7 


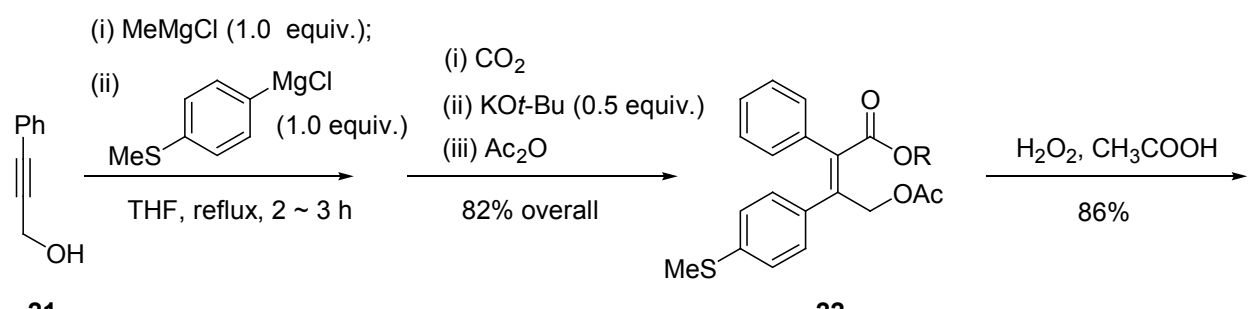

22<smiles>COS(=O)(=O)c1ccc(C(COC(C)=O)=C(C(=O)O)c2ccccc2)cc1</smiles>

23<smiles>[R]/C(=C\c1ccc(S(C)(=O)=O)cc1)C(=O)O</smiles>

$\mathrm{R}=4-\mathrm{NO}_{2} \mathrm{C}_{6} \mathrm{H}_{4}, 4-\mathrm{ClC}_{6} \mathrm{H}_{4}, \quad 2-\mathrm{ClC}_{6} \mathrm{H}_{4}, 4-\mathrm{CH}_{3} \mathrm{SO}_{2} \mathrm{C}_{6} \mathrm{H}_{4}, \mathrm{C}_{6} \mathrm{H}_{5}$, 1Naphthyl, 4- $\mathrm{CH}_{3} \mathrm{C}_{6} \mathrm{H}_{4}, 4-\mathrm{CH}_{3} \mathrm{SC}_{6} \mathrm{H}_{4}, 4-\mathrm{CH}_{3} \mathrm{OC}_{6} \mathrm{H}_{4}, 4-\mathrm{HOC}_{6} \mathrm{H}_{4}$, 4-Ph$\mathrm{CH}_{2} \mathrm{OC}_{6} \mathrm{H}_{4}, 4-\mathrm{H}_{2} \mathrm{NC}_{6} \mathrm{H}_{4}, 2,5-\left(\mathrm{CH}_{3} \mathrm{O}\right)_{2} \mathrm{C}_{6} \mathrm{H}_{4}, \mathrm{C}_{6} \mathrm{H}_{5} \mathrm{~S}, \mathrm{C}_{6} \mathrm{H}_{5} \mathrm{O}, \mathrm{CH}_{3}, \mathrm{H}$

图 3 化合物 II 通式

Figure 3 Formula of compound II

分析其原因可能是罗非昔布开环后对 COX-2 的选择性 有所降低. 结果显示. $\mathrm{II}_{1}, \mathbf{I I}_{3}, \mathrm{II}_{7}, \mathrm{II}_{\mathbf{9}}, \mathrm{II}_{10}, \mathbf{I I}_{13}, \mathbf{I I}_{15}, \mathbf{I I}_{16}$ 对小鼠耳肿胀模型表现显著的抗炎活性, $\mathbf{I I}_{1}, \mathbf{I I}_{13}, \mathbf{I I}_{15}$ 对 大鼠角叉菜胶致足跖肿胀模型显示较强的抗炎活性 ${ }^{[27]}$. 通过 Scheme 9 所示的化学转换, 可以合成系列罗非昔 布的代谢产物, 即罗非昔布的开环衍生物, 研究结果显 示对提高 COX-2 抑制活性效果不显著 ${ }^{[28]}$, 但可以指导 罗非昔布衍生物的合成.

\section{2 苯环修饰的衍生物}

通过比较可以发现化合物 4-[4-(甲磺酰基)苯基]-3环己基-2(5H) 呋喃酮(Scheme 10)是由罗非昔布 3 位上的 苯基换成环己基演变而来的. 苯环和环己基在电位上同 等的，可以预测与罗非昔布具有相似的药理活性 ${ }^{[29]}$.

由于 4 位苯环对位的取代基是必须的药效基团，对 其改造的报道不多，主要是利用生物电子等排原理来替 换甲磺酰基. 刘琨等 ${ }^{[30]} 2003$ 年报道了运用生物电子 等排原理用磺酰胺基来替换甲磺酰基合成了罗非昔布 衍生物 37 (Scheme 11), 经药理实验表明这种衍生物具 有与罗非昔布相似的止痛作用，且止痛作用无明显差 异，同时还具有一定的抗炎作用.

Habeeb 等 ${ }^{[31]}$ 于 2001 年报道了用叠氮来替换甲磺酰 基, 合成衍生物 41, 经活性测试表明其 $\mathrm{COX}-1 \mathrm{IC}_{50}=$ $159.7 \mu \mathrm{mol} / \mathrm{L} ; \mathrm{COX}-2 \mathrm{IC}_{50}=0.196 \mu \mathrm{mol} / \mathrm{L} ; \mathrm{COX}-2$ 的选 择性比罗非昔布大 11 倍. 本路线以对叠氮基苯乙酮为 原料，经三步反应制得，反应路线如 Scheme 12.

Navidpour 等 ${ }^{[32]}$ 于 2006 年设计合成了用三氮唑替

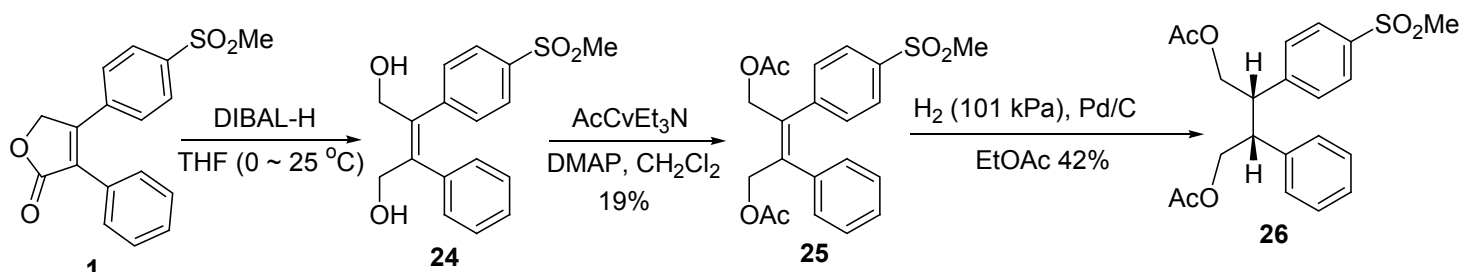

24

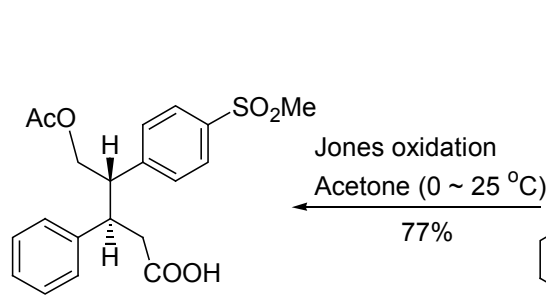

28

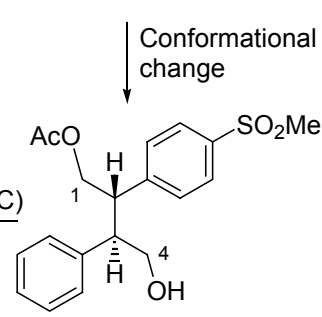

27

Scheme 9 


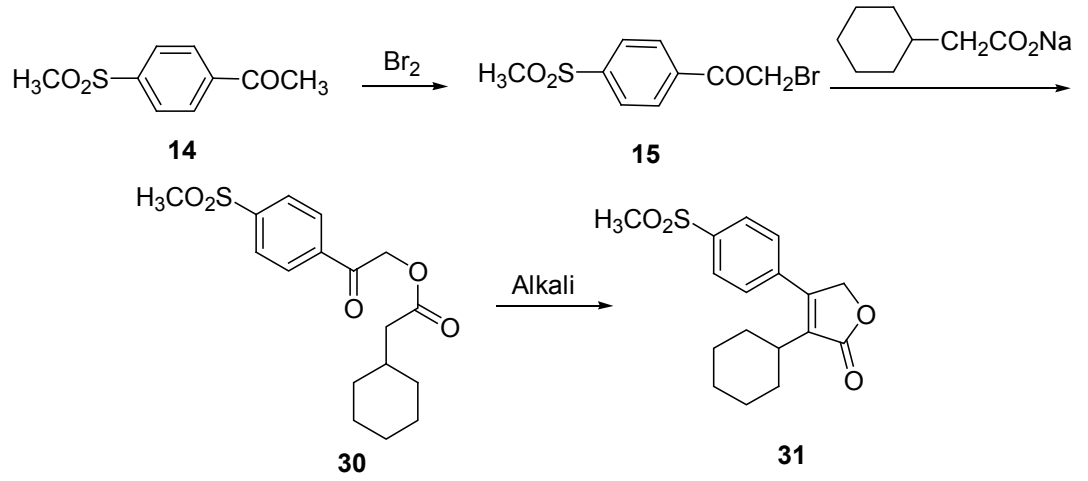

Scheme 10

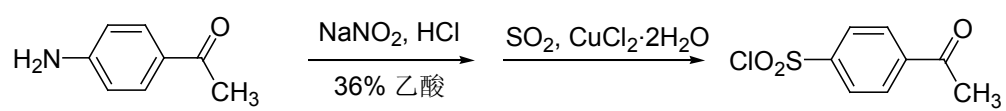

32

33
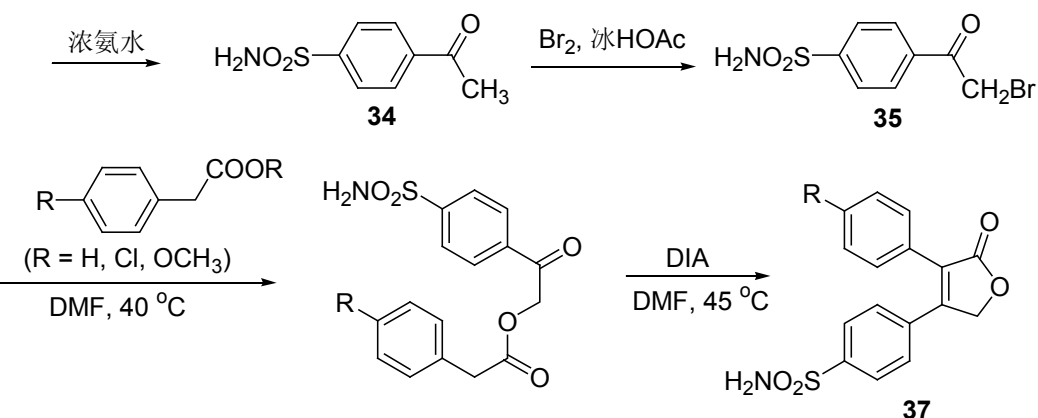

36

Scheme 11

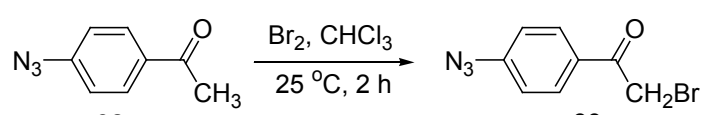

39

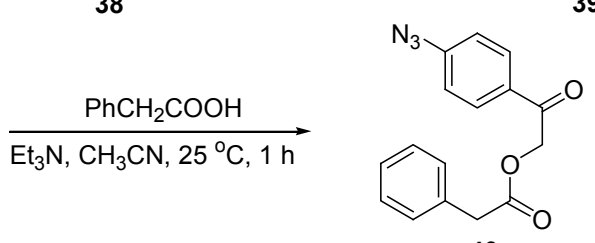

40

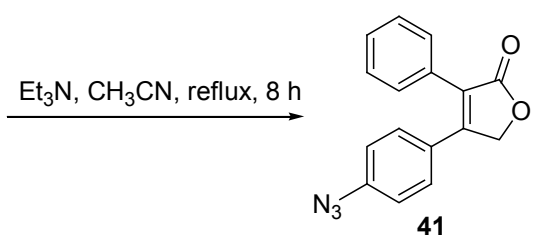

Scheme 12

换甲磺酰基的罗非昔布衍生物 45, 经分子模型表明与 罗非昔布相比此化合物可以更深的进入 COX-2 中与 $\operatorname{Arg} 513$ 发生静电作用, 展现出更好的 COX-2 抑制活性. 本路线以 $\alpha$-溴代-4-腈基-苯乙酥为原料, 经三步反应制 得, 反应路线 Scheme 13.

Zarghi 等 ${ }^{[33]} 2007$ 年报道了用甲磺酰胺基来替换甲 磺酰基，同时在 C-3 位苯环的对位连上各种取代基 $(\mathrm{H}, \mathrm{F}$,

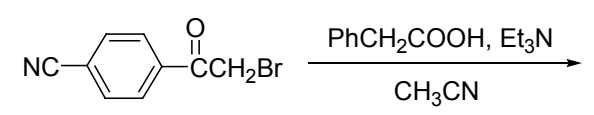

42<smiles>N#Cc1ccc(C(=O)COC(=O)Cc2ccccc2)cc1</smiles>

43<smiles>Cc1ccc(C2=C(c3ccccc3)C(=O)OC2c2ccc(-c3nn[nH]n3)cc2)cc1</smiles>

45

Scheme 13

$\mathrm{Cl}, \mathrm{Br}, \mathrm{Me}, \mathrm{OMe}$ ), 合成一系列的罗非昔布衍生物 51. 作 者通过活性测定发现甲磺酰胺基可以替换甲磺酰基，是 个合适的取代基团，同时 3 位苯环的对位连有 $\mathrm{H}, \mathrm{F}, \mathrm{Cl}$, $\mathrm{Br}$ 基团时兼有 COX-1, COX-2 的抑制作用，当 3 位苯环 对位连有 $\mathrm{Me}, \mathrm{OMe}$ 基团时化合物表现出 COX-2 的选择 性抑制作用. 路线以对氨基苯乙酮为原料经四步反应制 
得一系列衍生物 51, 反应路线如 Scheme 14 .

Chen 等 $^{[34]} 2006$ 年报道了罗非昔布 C-3 位苯环对位 改造的产物, 作者希望寻找到具有 COXs 和 LOXs 双重 抑制活性的化合物. 通过体外 COX-1/COX-2 和 5-LOX/15-LOX 异构酶活性测试, 篎选出了两种活性较 好的化合物 58, 59. 通过进一步的体内活性试验, 发现 这两种化合物比传统的 5-LOX 酶抑制剂咖啡酸和 15-LOX 酶抑制剂去甲二氢愈创木酸活性要高，但是 COX-2 酶抑制活性没有罗非昔布高. 本研究发现罗非 昔布 C-3 位苯环对位引入肜基是设计合成具有 COXs 和 LOXs 双重抑制活性化合物的合适模板. 反应路线如 Scheme 15.

Caturla 等 ${ }^{[35]} 2006$ 年报道了用手性的甲基亚砜取代 C-4 苯环对位消旋的甲基砜, 设计出了一种罗非昔布的 前药 63. 该化合物在体内转化为原药罗非昔布起到抗 炎镇痛作用. 由于亚砜基比砜基具有更好溶解性，所以 与原药罗非昔布相比, 其具有更好的药代动力学水平及 更好的药理学应答. 反应路线如 Scheme 16. Smith 等 ${ }^{[36]}$ 研究设计了一种水溶性的前药, 进入体内后转化为原药
起作用, 此类前药容易进入血脑屏障, 可以通过静脉给 药到达脑部, 起到治疗中风的作用. 作者选取了 C-3 位 苯环上含氟原子取代的罗非昔布衍生物为研究对象, 设 计出系列前药 71. 经过活性测试, 笁选出化合物 72 为 理想的前药, 通过静脉给药, 在体内迅速转化为原药到 达脑部起作用. 反应路线如 Scheme 17.

为了适合静脉给药, 增加药物的水溶性, 2003 年 Black 等 ${ }^{[37]}$ 设计合成了 C-5 位含有烷氧基取代的罗非昔 布衍生物 76. 这类衍生物在 $\mathrm{NaOH}$ 作用下生成水溶性的 钠盐, 作为前药适用于静脉给药, 在体内转化为原药, 增加了药物的生物利用度. 通过对这一系列衍生物的药 理活性测试, 发现 C-3 苯环对位氯原子取代效果较好, 笁选出化合物 77b. 反应路线如 Scheme 18.

2004 年 Zarghi 等 ${ }^{[38]}$ 设计合成了在 C-3, C-4 位苯环 对位分别被 $\mathrm{SO}_{2} \mathrm{NH}_{2}, \mathrm{SO}_{2} \mathrm{NHCOMe}$ 或 $\mathrm{SO}_{2} \mathrm{~N}_{3}$ 取代的罗 非昔布衍生物, 共计 6 种, 用来研究这些基团对活性的 影响. 通过活性测试, 笁选出化合物 82b 具有较好的选 择性 COX-2 酶抑制活性. 通过分子模型表明化合物 82b 能更好的与 COX-2 酶结合. 反应路线如 Scheme 19.

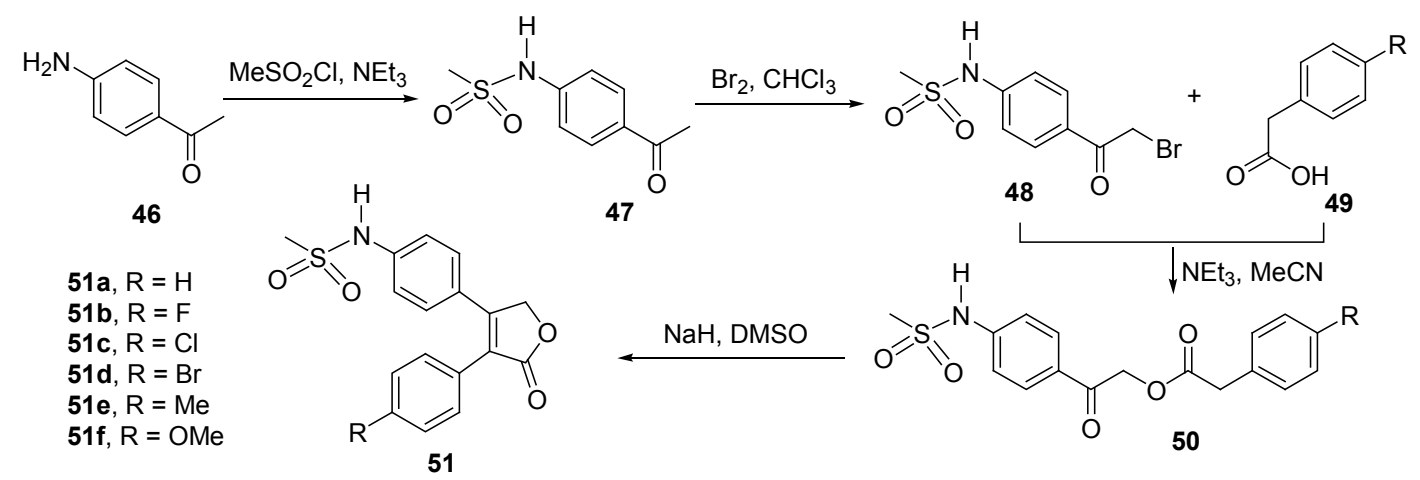

Scheme 14

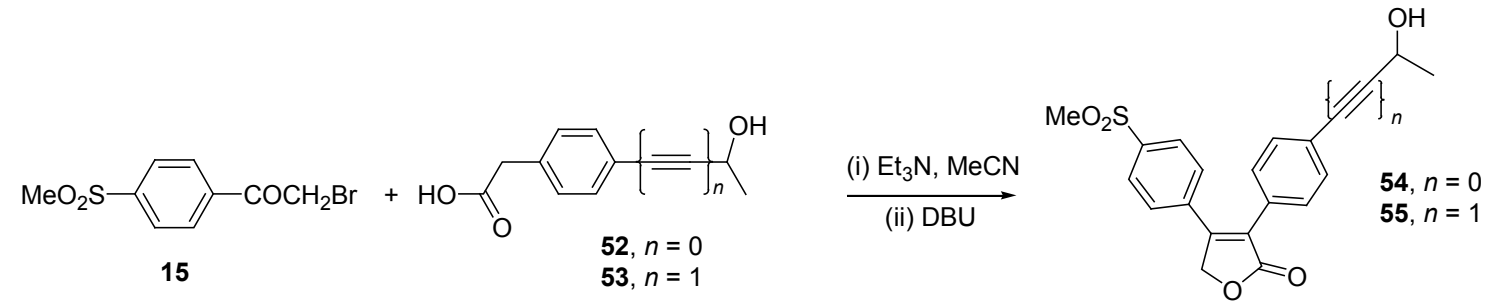

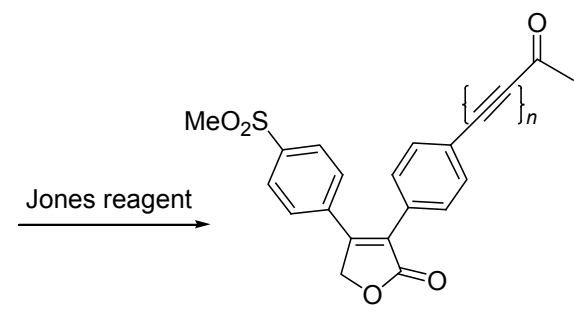

$56, n=0$

$57, n=1$
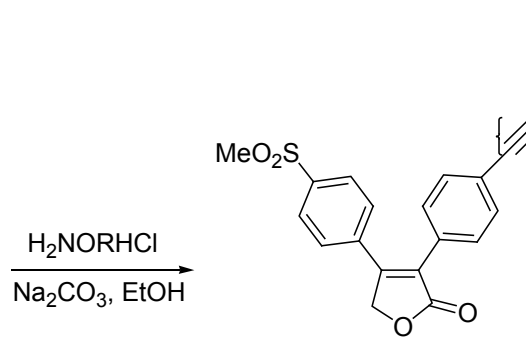

$58, n=0$

$\mathbf{5 9}, n=1$

Scheme 15 
<smiles>CS(=O)(=O)c1ccc(C(=O)CBr)cc1</smiles>

(a) Ti(Oi-Pr) $)_{4} /(R, R)$-DET ( $\left.V: V=1: 4\right), t-\mathrm{BuOOH}, 1,2-\mathrm{DCE}$ (b) Ti(Oi-Pr) $)_{4} /(S, S)$-DET $(V: V=1: 4), t$-BuOOH, 1,2-DCE<smiles>C[S+]([O-])([O-])c1ccc(C2=C(c3ccccc3)C(=O)OC2)cc1</smiles>

Scheme 16<smiles>CC(C)=C(C)C(=O)Cc1ccc(F)c(F)c1</smiles><smiles>CC(=O)OCC(=C(CO)c1ccc(F)c(F)c1)c1ccc(S(C)(=O)=O)cc1</smiles>

68<smiles>[Y]c1ccc(C2=C(c3ccc(S(C)(=O)=O)cc3)COC2=O)cc1F</smiles><smiles>CC(=O)OC/C(=C(/C(=O)O)c1ccc(S(C)(=O)=O)cc1)c1ccc(F)c(F)c1</smiles>

69<smiles>CC(=O)OCC(=C(C(=O)OCC[N+](C)(C)C)c1ccc(S(C)(C)C)cc1)c1ccc(F)c(F)c1</smiles>

70<smiles>CC(=O)OCC(=C(C(=O)OCC[N+](C)(C)C)c1cccc(F)c1)c1ccc(S(C)(=O)=O)cc1</smiles>

72

Scheme 17

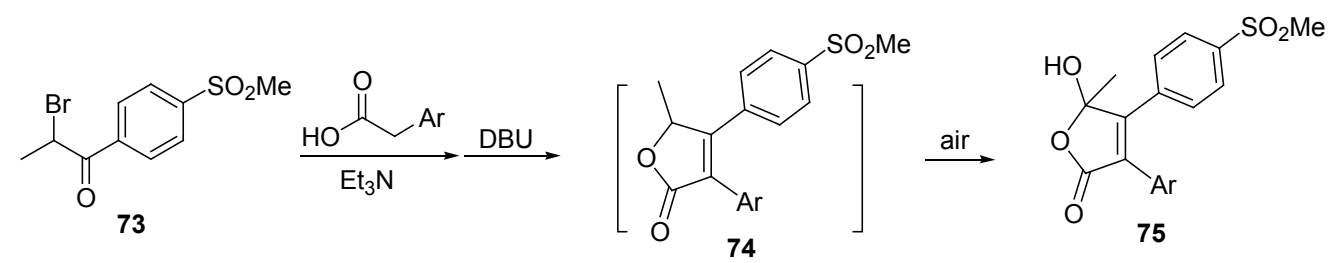<smiles>CO[R]([O-])(O)c1ccc(C2=C([Al])C(=O)OC2(C)O)cc1</smiles>

76<smiles>[R]c1ccc(C2=C(c3ccc(S(C)(=O)=O)cc3)C(C)(O)OC2=O)cc1</smiles>

$76 a, \mathrm{R}=\mathrm{H}$

76b, $\mathrm{R}=\mathrm{Cl}$

Scheme 18

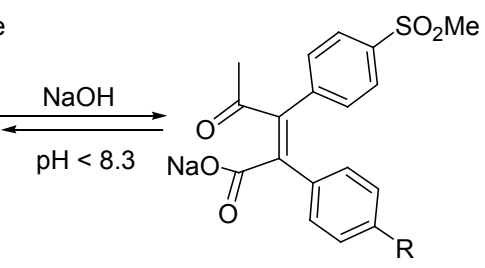

77a, $\mathrm{R}=\mathrm{H}$ $77 \mathrm{~b}, \mathrm{R}=\mathrm{Cl}$ 
<smiles>[R]c1ccc(C(=O)CBr)cc1</smiles>

78

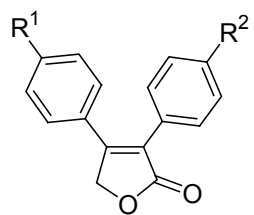

80a, $\mathrm{R}^{1}=\mathrm{SO}_{2} \mathrm{Me}, \mathrm{R}^{2}=\mathrm{H}$

80b, $\mathrm{R}^{1}=\mathrm{H}, \mathrm{R}^{2}=\mathrm{SO}_{2} \mathrm{Me}$<smiles>[R]c1ccc(CC(=O)O)cc1</smiles>

79

$\mathrm{ClSO}_{3} \mathrm{H}$
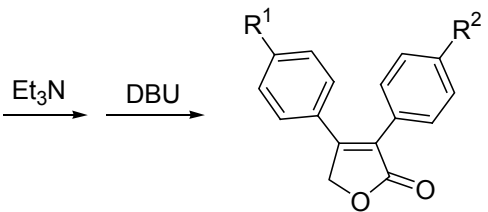

80a, $\mathrm{R}^{1}=\mathrm{SO}_{2} \mathrm{Me}, \mathrm{R}^{2}=\mathrm{H}$ 80b, $\mathrm{R}^{1}=\mathrm{H}, \mathrm{R}^{2}=\mathrm{SO}_{2} \mathrm{Me}$

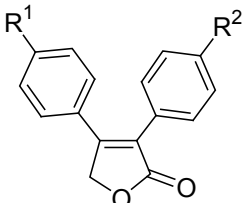

81a, $R^{1}=\mathrm{SO}_{2} \mathrm{Me}, \mathrm{R}^{2}=\mathrm{SO}_{2} \mathrm{NH}_{2}$

81b, $\mathrm{R}^{1}=\mathrm{SO}_{2} \mathrm{NH}_{2}, \mathrm{R}^{2}=\mathrm{SO}_{2} \mathrm{Me}$

82a, $\mathrm{R}^{1}=\mathrm{SO}_{2} \mathrm{Me}, \mathrm{R}^{2}=\mathrm{SO}_{2} \mathrm{NHCOMe}$

82b, $R^{1}=\mathrm{SO}_{2} \mathrm{NHCOMe}, \mathrm{R}^{2}=\mathrm{SO}_{2} \mathrm{Me}$

83a, $R^{1}=\mathrm{SO}_{2} \mathrm{Me}, \mathrm{R}^{2}=\mathrm{SO}_{2} \mathrm{~N}_{3}$

83b, $\mathrm{R}^{1}=\mathrm{SO}_{2} \mathrm{~N}_{3}, \mathrm{R}^{2}=\mathrm{SO}_{2} \mathrm{Me}$

\section{Scheme 19}

2005 年 Weber 等 ${ }^{[39]}$ 保留五元内酯环, 改变两个苯环 的位置，同时引入羟基来设计合成了一系列衍生物 $\mathbf{8 9}$, 95, 以期篮选出具有更好药理活性的化合物. 作者通过 两种抗炎模型活性测试对比, 发现 $89 \mathrm{~g}, 95 \mathrm{f}$ 具有很好的 抗炎活性, 下一步对这两种化合物进行深入的药理学和 毒理学研究. 反应路线如 Scheme 20.

罗非昔布的 5 位极易被氧化, 在空气中即能被氧 化. 氧化的结果是 5 位接上一个羟基, 此步氧化对罗 非昔布的体内代谢非常重要，因为羟基和体内的葡 萄糖醛酸结合, 使药物能够很快的排出体外. 体内代 谢反应式如 Scheme 21 所示, Scheme 22 所示是化学法 氧化得到 5 位被羟基化的产物 ${ }^{[0]}$, 通过改变羟基的位 置丰富了罗非昔布衍生物的数量.

专利 ${ }^{[41]}$ 报道 3 位苯环具有双取代基的系列罗非昔布 衍生物的合成, 以及该系列化合物在抗炎及 COX-2 介 导的疾病中的作用. 图 4 通式所示系列化合物的 COX-2 抑制活性是 COX-1 抑制活性的 100 倍. 所有化合物的 $\mathrm{COX}-2$ 抑制活性 $\mathrm{IC}_{50}$ 值为 $1 \mathrm{nmol} / \mathrm{L}$ 到 $1 \mu \mathrm{mol} / \mathrm{L}$. 而布洛 芬对 COX-2 的抑制活性为 $\mathrm{IC}_{50}$ 为 $1 \mu \mathrm{mol} / \mathrm{L}$, 吲哚美辛对 $\mathrm{COX}-2$ 的抑制活性为 $\mathrm{IC}_{50}$ 为 $100 \mathrm{nmol} / \mathrm{L}$. 对合成的化合 物进行了典型的角叉菜胶致大鼠足跖肿胀模型评价其 抗炎活性, 结果显示该系列衍生物 IV 具有良好的抗炎 活性. 文献[42]评估了 MF-tricyclic $\left(\mathrm{R}^{1}=\mathrm{F}, \mathrm{R}^{2}=\mathrm{F}\right)$ 对常 用的四个种属, 即小鼠, 大鼠, 豚鼠和兔的选择性 COX-2 抑制活性. 在豚鼠和兔全血分析中, 该化合物抑 制脂多糖诱导的 PGE2 生成的 $\mathrm{IC}_{50}$ 分别为 0.6 和 2.8 $(\mathrm{COX}-2) \mu \mathrm{mol} / \mathrm{L}$. 相比之下，该化合物对凝块诱导的血. 栓的形成显示一个非常弱的活性, $\mathrm{IC}_{50}$ 值分别为对
(COX-1) 10 (豚鼠)和 23 (兔) $\mu \mathrm{mol} / \mathrm{L}$. 对大鼠和小鼠试验 也显示了良好的生物活性.

文献[43]报道了系列化合物 $\mathbf{V}$ (图 5)的合成及其选 择性 COX-2 抑制活性, 其中化合物 $101\left(\mathrm{IC}_{50} \mathrm{COX}-1 \geqslant\right.$ $\left.100 \mu \mathrm{mol} / \mathrm{L}, \mathrm{IC}_{50} \mathrm{COX}-2=2 \mu \mathrm{mol} / \mathrm{L} ; \mathrm{SI}>50\right)$. 分子对接 研究显示化合物 101 可以到达 COX-2 的结合位点, 从而 为其选择性 COX-2 抑制活性提供分子基础.

文献[44]报道了系列 VI, VII, VIII(图 6)含有二芳杂 环的新型罗非昔布衍生物的合成，并报道了他们的 COX-1, COX-2 抑制活性. 系列 VI 中化合物 $\mathbf{1 0 2}(\mathrm{COX}-1$ $\mathrm{IC}_{50}=0.061 \mu \mathrm{mol} / \mathrm{L} ; \mathrm{COX}-2 \quad \mathrm{IC}_{50}=0.325 \mu \mathrm{mol} / \mathrm{L} ; \mathrm{SI}=$ 0.19)为最好的 COX 抑制剂. 系列 VII 中化合物 103 为 最好的 COX-2 抑制剂, COX-2 抑制活性为罗非昔布的 38 倍(103: COX-2 $\mathrm{IC}_{50}=0.011 \mu \mathrm{mol} / \mathrm{L}$; 罗非昔布: 0.398 $\mu \mathrm{mol} / \mathrm{L}$ )，但是对 COX-2 没有选择抑制活性. 化合物 104 表现出强的选择性 COX-2 抑制活性(COX-1 $\mathrm{IC}_{50}=1$ $\left.\mu \mathrm{mol} / \mathrm{L}, \mathrm{COX}-2 \mathrm{IC}_{50}=0.011 \mu \mathrm{mol} / \mathrm{L} ; \mathrm{SI} \approx 92\right)$, 分子对接 研究发现化合物 102 和 103 可以伸入 COX-1 和 COX-2 的结合位点因而具有 COX 抑制活性.

文献[45]报道了一种新型的混合释放 NO 的抗炎内 酯前药(NONO-coxib 106)及母体药物 105 的合成(图 7). 前药内酯 106 是一个选择性 COX-2 抑制剂表现出良好 的抗炎活性 $\left(E D_{50}=72.2 \mathrm{mmol} / \mathrm{kg} \mathrm{po}\right)$, 其抗炎活性介于 塞来昔布 $\left(\mathrm{ED}_{50}=30.9 \mu \mathrm{mol} / \mathrm{kg}\right.$ po $)$ 和布洛芬 $\left(\mathrm{ED}_{50}=327\right.$ $\mu \mathrm{mol} / \mathrm{kg} \mathrm{po})$ 之间.

文献[46]研究了 COX-2 抑制剂的混合苂光结合物的 合成, 试图寻找对 COX-2 酶的肿瘤标志物. 其中合成了 一个罗非昔布衍生物 107 , 该化合物表现出了一定的选 


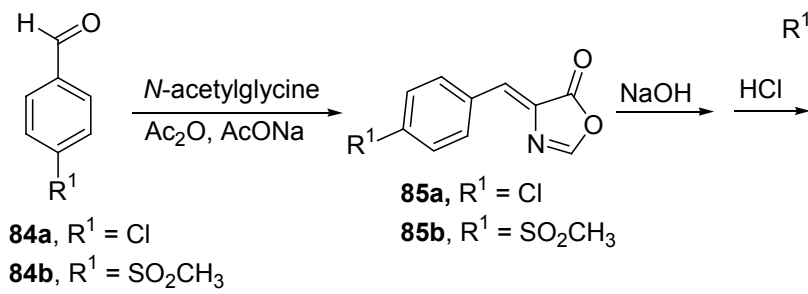<smiles>[R]c1ccc(C(=O)c2ccccc2)cc1</smiles>

88<smiles>[R]c1ccc(C2=C(O)C(=O)OC2c2ccc([R])cc2)cc1</smiles>

89 86a, $\mathrm{R}^{1}=\mathrm{Cl}$ 86b, $\mathrm{R}^{1}=\mathrm{SO}_{2} \mathrm{CH}_{3}$ $86 c, R^{1}=H$

87a, $\mathrm{R}^{1}=\mathrm{Cl}$

87b, $\mathrm{R}^{1}=\mathrm{SO}_{2} \mathrm{CH}_{3}$ $87 \mathrm{c}, \mathrm{R}^{1}=\mathrm{H}$

$86 d, R^{1}=O H$<smiles>[R]C(=O)C(O)=Cc1ccc([I-])cc1</smiles>

$87 d, R^{1}=\mathrm{OH}$

$89 a, \mathrm{R}^{1}=\mathrm{H} ; \mathrm{R}^{2}=\mathrm{SO}_{2} \mathrm{CH}_{3}$

89b, $\mathrm{R}^{1}=\mathrm{SO}_{2} \mathrm{CH}_{3} ; \mathrm{R}^{2}=\mathrm{H}$

$89 \mathrm{c}, \mathrm{R}^{1}=\mathrm{Cl} ; \mathrm{R}^{2}=\mathrm{SO}_{2} \mathrm{CH}_{3}$

89d, $\mathrm{R}^{1}=\mathrm{SO}_{2} \mathrm{CH}_{3} ; \mathrm{R}^{2}=\mathrm{Cl}$

$\mathrm{Pd} / \mathrm{C}, \mathrm{H}_{2} 89 \mathrm{e}, \mathrm{R}^{1}=\mathrm{OH} ; \mathrm{R}^{2}=\mathrm{SO}_{2} \mathrm{CH}_{3}$

$89 f, R^{1}=\mathrm{SO}_{2} \mathrm{CH}_{3} ; \mathrm{R}^{2}=\mathrm{OBn}$

$89 \mathrm{~g}, \mathrm{R}^{1}=\mathrm{SO}_{2} \mathrm{CH}_{3} ; \mathrm{R}^{2}=\mathrm{OH}$

\footnotetext{
$\mathrm{R}^{2} \bigcap_{\mathrm{O}}^{\mathrm{OH}} \stackrel{\mathrm{SnCl}_{2}, \mathrm{EtOH}}{\longrightarrow} \mathrm{R}^{2} \bigwedge_{\mathrm{O}}^{\mathrm{OEt}} \stackrel{\mathrm{Br}_{2}}{\longrightarrow} \mathrm{R}^{2} \bigwedge_{\mathrm{O}}^{\mathrm{Br}} \mathrm{OEt}$

90a, $\mathrm{R}^{2}=\mathrm{C}_{6} \mathrm{H}_{5}$

90b, $\mathrm{R}^{2}=4-\mathrm{SO}_{2} \mathrm{CH}_{3} \mathrm{C}_{6} \mathrm{H}_{5}$

91a, $\mathrm{R}^{2}=\mathrm{C}_{6} \mathrm{H}_{5}$

92a, $\mathrm{R}^{2}=\mathrm{C}_{6} \mathrm{H}_{5}$

90c, $\mathrm{R}^{2}=4-\mathrm{ClC}_{6} \mathrm{H}_{5}$

91b, $\mathrm{R}^{2}=4-\mathrm{SO}_{2} \mathrm{CH}_{3} \mathrm{C}_{6} \mathrm{H}_{5}$

91c, $\mathrm{R}^{2}=4-\mathrm{ClC}_{6} \mathrm{H}_{5}$

92b, $\mathrm{R}^{2}=4-\mathrm{SO}_{2} \mathrm{CH}_{3} \mathrm{C}_{6} \mathrm{H}_{5}$

92c, $\mathrm{R}^{2}=4-\mathrm{ClC}_{6} \mathrm{H}_{5}$
}

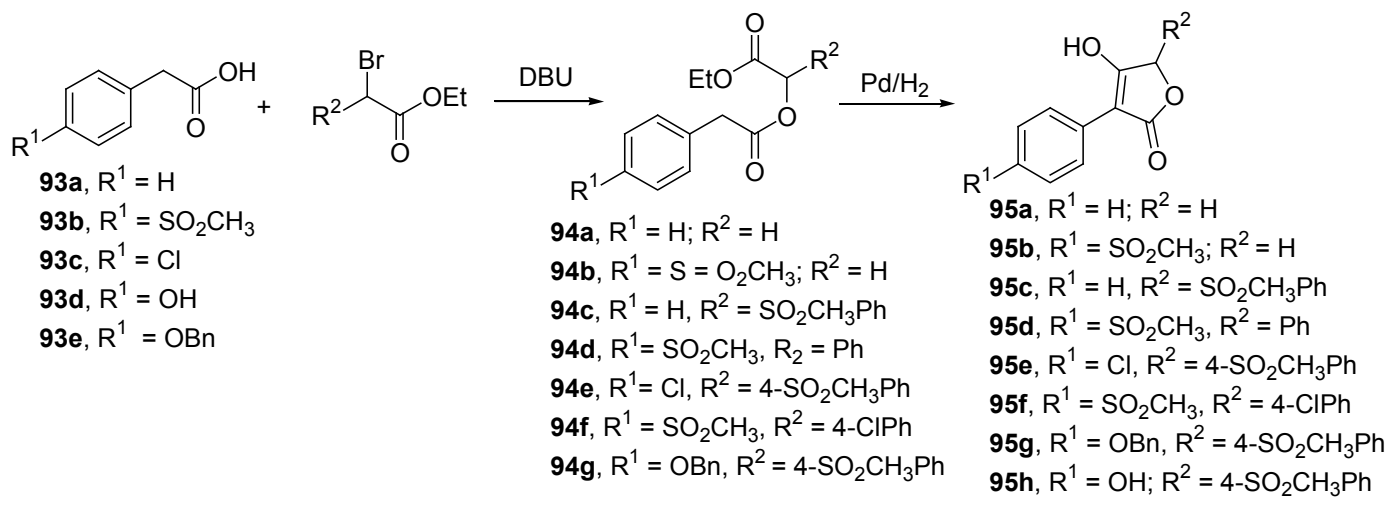

Scheme 20<smiles>COc1ccc(C2=C(c3ccccc3)C(=O)OC2)cc1</smiles>

1 (Rofecoxib)

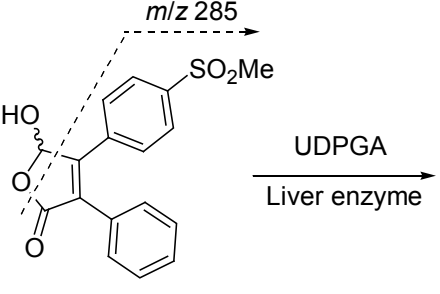

96

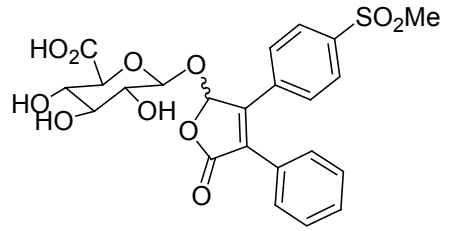

97

Scheme 21

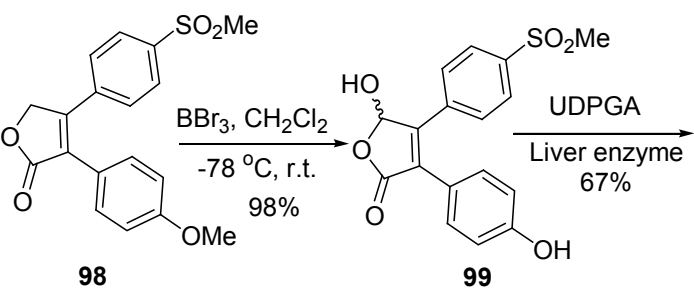

98<smiles>COS(=O)(=O)c1ccc(C2=C(c3ccc4c(c3)C(O)(C(O)(O)O)C(O)C4O)C(=O)OC2)cc1</smiles>

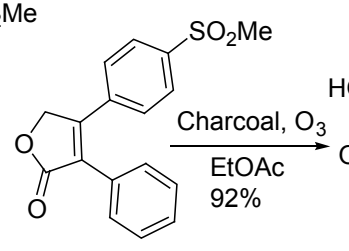

1 (Rofecoxib)

Scheme 22 
<smiles>[R]c1ccc(C2=C(c3ccc(S(C)(=O)=O)cc3)COC2=O)cc1[R]</smiles>

$\mathrm{R}^{1}=\mathrm{F}, \mathrm{Cl}, \mathrm{Br}, \mathrm{H} ; \mathrm{R}^{2}=\mathrm{F}, \mathrm{Cl}, \mathrm{Br}, \mathrm{H}$

图 4 化合物 IV 通式

Figure 4 Formula of compound IV<smiles>[R]c1ccc(-c2oc(=O)n([R])c2-c2ccccc2)cc1</smiles>

图 5 化合物 $\mathbf{V}$ 通式

Figure 5 Formula of compound $\mathbf{V}$<smiles>[R]c1ccc(C2=C(c3ccc4c(c3)oc(=O)n4C)COC2=O)cc1C1=C(c2ccc3c(c2)oc(=O)n3C)COC1=O</smiles>

图 6 化合物 VI, VII, VIII 通式

Figure 6 Formula of compounds VI, VII and VIII

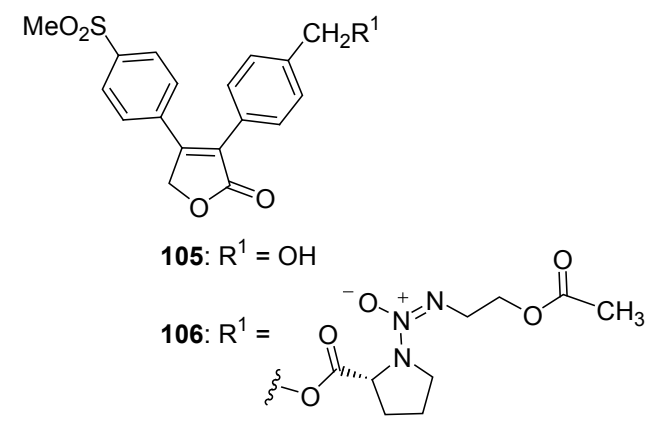

图 7 化合物 105 和 106 的结构

Figure 7 The structures of compounds $\mathbf{1 0 5}$ and $\mathbf{1 0 6}$

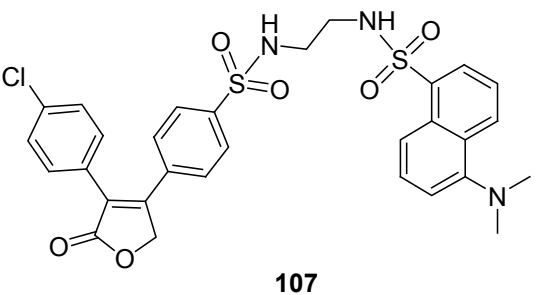

图 8 化合物 107 的结构

Figure 8 The structure of compound $\mathbf{1 0 7}$
择性 COX-2 抑制活性(COX-1 $\mathrm{IC}_{50}>100 \mu \mathrm{mol} / \mathrm{L}$; COX-2 $\left.\mathrm{IC}_{50}=7.2 \mu \mathrm{mol} / \mathrm{L} ; \mathrm{SI}>14.0\right)$.

\section{3 总结及展望}

本文介绍了近年来罗非昔布合成工艺的研究以及 罗非昔布衍生物的研究取得的一些成就. 罗非昔布合成 工艺已经成熟，基本可以达到原料廉价易得，操作方便， 条件温和, 污染相对较少的要求. 系列衍生物的合成在 选择性 COX-2 抑制活性及抗炎活性方面取得了良好的 效果，为开发新的非甾体抗炎药奠定了基础，同时也为 以 COX-2 为靶标的活性化合物的设计合成开辟了思路. 期待抗炎活性好且毒副作用小的罗非昔布衍生物真正 成为药物分子被开发上市，为人类造福.

\section{References}

[1] Wang, R. -Y. Chin. J. New Drugs 2000, 9, 661 (in Chinese). (王仁云，中国新药杂志, 2000, 9, 661.)

[2] Scott, L. J.; Lamb, H. M. Drugs 1999, 58, 499.

[3] Ehrich, E. W.; Dallob, A.; Lepeleire, I. D.; Hecken, A. V.; Riendeau, D.; Yuan, W.; Porras, A.; Wittreich, J.; Seibold, J. R.; Schepper, P. D.; Mehlisch, D. R.; Gertz, B. J. Clin. Pharmacol. Ther. 1999, 65, 336

[4] Hawkey, C. J. Lancet 1999, 353, 307.

[5] Hecken, A. V.; Depré, M.; Ehrich, E.; Lepeleire, I. D.; Hilliard, D.; Porras, A.; Wong, P.; Buntinx, A.; Gertz, B. J.; Schepper, P. J. D. Clin. Pharmacol. Ther. 1999, 65, 164.

[6] Morrison, B. W.; Daniels, S. E.; Kotey, P. Obstet. Gynecol. 1999, 94, 504

[7] Zhang, W. China Pharmacist 2005, 8, 474 (in Chinese). (张伟，中国药师, 2005, 8, 474.)

[8] Shrn, J; Li, B.-B. Evaluation Anal. Drug 2006, 6, 69 (in Chinese) (沈娟, 李涁涁, 中国医院用药评价与分析, 2006, 6, 69.)

[9] Ma, Y.-Z.; Liu, Y.-X.; Zhou, Y.-P.; Mei, W.-J.; She, Z.-G. J. Guandong College Pharm. 2005, 21, 649 (in Chinese). (马玉卓, 刘鹰翔, 周玉平, 梅文杰, 余志刚, 广东药学院学报, 2005, 21, 649.)

[10] Desmond, R.; Dolling, U.; Marcune, B.; Tillyer, R.; Tschaen, D. WO 9608482, 1996 [Chem. Abstr. 1996, 125, 86474].

[11] Atkinson, J. G., Wang, Z.-Y. WO 9613483, 1996 [Chem. Abstr. 1996, 125, 114294].

[12] Desmond, R.; Dolling, U. H.; Frey, L. F.; Tillyer, R.; Tschaen, D. M. WO 9800416, 1996 [Chem. Abstr. 1996, 128, 114869]

[13] Michel, T.; Jacques, Y. G.; Yves, L.; Serge, L.; Hélène, P.; Petpiboon, P.; Wang, Z.-Y. Synthesis 2001, 1778.

[14] Ao, G. Z.; Wang, W. D.; Zhang, Y. H. Chin. J. Pharm. 2002, 6, 267 (in Chinese).

(敖桂珍，王未东，张奕华，中国医药工业杂志, 2002, 6, 267.)

[15] Gans, K. R.; Galbrainth, W.; Roman, R. J.; Haber, S. B.; Kerr, J. S.; Schmidt, W. K.; Smith, C.; Hewes, W. E.; Ackerman, N. R. J Pharmacol. Exp. Ther. 1990, 254, 180.

[16] Magolda, R. L.; Batt, D.; Covington, M. B. Inflamm. Res. 1995, 44 (suppl. 3), A274.

[17] Penning, T. D.; Talley, J. J.; Bertenshaw, S. R.; Carter, J.S.; Collins, P. W.; Docter, S.; Graneto, M. J.; Lee, L. F.; Malecha, J. W.; Miyashiro, J. M.; Rogers, R. S.; Rogier, D. J.; Yu, S. S.; Anderson, G. D.; Burton, E. G.; Cogburn, J. N.; Gregory, S. A.; 
Koboldt, C. M.; Perkins,W.E.; Seibert, K.; Veenhuizen, A. W.; Zhang, Y. Y.; Isakson, P. C. J. Med. Chem. 1997, 40, 1347.

[18] Reitz, D. B.; Li, J. J.; Norton, M. B.; Reinhard, E. J.; Collins, J. T.; Anderson, G. D.; Gregory, S. A.; Koboldt, C. M.; Perkins,W. E. J. Med. Chem. 1994, 37, 3878.

[19] Vane, J. R.; Botting, R. M. Inflamm. Res. 1995, 44, 1.

[20] Chan, C. C.; Boyce, S.; Brideau, C.; Charleson, S.; Cromlish, W.; Ethier, D.; Evans, J.; Ford-Hutchinson, A. W.; Forrest, M. J.; Gauthier, J. Y.; Gordon, R.; Gresser, M.; Guay, J.; Kargman, S.; Kennedy, B.; Leblanc, Y.; Leger, S.; Mancini, J.; O'Neill, G. P.; Ouellet, M.; Patrick, D.; Percival, M. D.; Perrier, H.; Prasit, P.; Rodger, I. J. Pharmacol. Exp. Ther. 1999, 290, 551.

[21] Tacconelli, S.; Capone, M. L.; Sciulli, M. G.; Ricciotti, E.; Patrignani, P. Curr. Med. Res. Opin. 2002, 18, 503.

[22] Katz, J.; Fry, D.; Kehlet, H. 22nd Annual Scientific Meeting of the American Pain Society, Chicag, 2003, pp. 88 89.

[23] Su, X.-Y.; Wang, E.-H. Prog. Pharmacel. Sci. 2004, 28, 309 (in Chinese). (苏晓岳, 王尔华, 药学进展, 2004, 28, 309.)

[24] Zhao, L.-Q.; Zhang, S.-F. Chin. J. Med. Chem. 1999, 9, 138 (in Chinese).

(赵丽琴, 张守芳, 中国药物化学杂志, 1999, 9, 138.)

[25] Saeed, E.; Alireza, F. Chin. J. Chem. 2006, 24, 791.

[26] Engelhardt, F. C.; Shi, Y.-J.; Cowden, C. J.; Conlon, D. A.; Pipik, B.; Zhou, G.; McNamara, J. M.; Dolling. U. J. Org. Chem. 2006, 71,480 .

[27] Ao, G.-Z.; Zhang, Y.-H.; Ji, H.; Deng, G.; Wu, Y.-J.; Peng, S.-X. J. China Pharm. Univ. 2002, 33, 460 (in Chinese).

(敖桂珍, 张奕华, 季晖, 邓钢, 吴艳俊, 彭司勋, 中国药科大学 学报, 2002, 33, 460.)

[28] Wang, F.-Y.; Li, S.-L.; Feng, W.-L. J. Beijing Univ. Chem. Technol. 2004, 31, 84 (in Chinese).

(汪风顶, 李顺来, 冯文林, 北京化工大学学报, 2004, 31, 84).

[29] Pan, H.-G.; Yu, X.-H.; Wu, D.-J. Synth. Chem. 2001, 9, 265 (in Chinese) (潘海港, 虞金红, 吴达俊, 合成化学, 2001, 9, 265.)

[30] Liu, K. M.S. Thesis, Hebei Medical University, Shijiazhuang, 2003 (in Chinese). (刘琨, 硕士论文, 河北医科大学, 石家庄, 2003.)

[31] Habeeb, A. G.; Praveen Rao, P. N.; Knaus, E. E. J. Med. Chem. 2001, 44, 3039.
[32] Navidpour, L.; Amini, M.; Shafaroodi, H.; Abdi, k.; Ghahremani, M. H.; Dehpour, A. R.; Shafiee, A. Bioorg. Med. Chem. Lett. 2006, 16, 4483.

[33] Zarghi, A.; Praveen Rao P. N.; Knaus, E. E. Bioorg. Med. Chem. 2007, 15, 1056.

[34] Chen, Q.-H.; Praveen, Rao P. N. Knaus, E. E. Bioorg. Med. Chem. 2006, 14, 7898.

[35] Caturla, F.; Amat, M.; Reinoso, R. F.; Cordoba, M.; Warrellow, G. Bioorg. Med. Chem. Lett. 2006, 16, 3209.

[36] Smith, N. D.; Reger, T. S.; Payne, J.; Zunic, J.; Lorrain, D.; Correa, L.; Stock, N.; Cramer, M.; Chen, W.-C.; Yang, J.; Prasit, P.; Munoz, B. Bioorg. Med. Chem. Lett. 2005, 15, 3197.

[37] Black, W. C.; Brideau, C.; Chan, C.-C.; Charleson, S.; Cromlish, W.; Gordon, R.; Grimm, E. L.; Hughes, G.; Leger, S.; Li, C.-S.; Riendeau, D.; Therien, M.; Wang, Z.-Y.; Xu, L.-J.; Prasit, P. Bioorg. Med. Chem. Lett. 2003, 13, 1195.

[38] Zarghi, A.; Praveen Rao P. N.; Knaus, E. E. Bioorg. Med. Chem. Lett. 2004, 14, 1957.

[39] Weber, V.; Rubat, C.; Duroux, E.; Lartigue, C.; Michel, M.; Pascal, C. Bioorg. Med. Chem. 2005, 13, 4552.

[40] Nicoll-Grith, D. A.; Yergey, J. A.; Trimble, L. A.; Silva, J. M.; Li, C.; Chauret, N.; Gauthier, J. Y.; Grimm, E.; Leger, S.; Roy, P.; Therien, M.; Wang, Z. Y.; Prasit, P.; Zamboni, R.; Young, R. N.; Brideau, C.; Chan, C.-C.; Mancini, J.; Riendeau, D. Bioorg. Med. Chem. Lett. 2000, 10, 2683.

[41] Desmond, R.; Dolling, U.; Marcune, B.; Tillyer, R.; Tschaen, D. WO 9608482, 1996 [Chem. Abstr. 1996, 125, 86474].

[42] Rowland, S. E.; Clark, P.; Gordon, R.; Mullen, A. K.; Guay, J.; Dufresne, L.; Brideau, C.; Cote, B.; Ducharme, Y.; Mancini, J.; Chan, C.-C.; Audoly, L.; Xu, D. Eur. J. Pharmacol. 2007, 560, 216.

[43] Dundar, Y.; Ünlü, S.; Banoglu, E.; Entrena, A.; Costantino, G.; Nuñez, M. T.; Ledo, F.; Sahin, M. F.; Noyanalpan, N. Eur. J. Med. Chem. 2009, 44,1830.

[44] Eren,G.; Ünlü, S.; Nuñez, M. T.; Labeaga,L.; Ledo, F.; Entrena, A.; Banoğlu, E.; Costantino, G.; Sahin. M. F. Bioorg. Med. Chem. 2010, 18, 6367.

[45] Abdellatif, K. R. A.; Huang, Z. J.; Chowdhury, M. A.; Kaufman, S.; Knaus, E.E. Bioorg. Med. Chem. Lett.2011, 21, 3951.

[46] Bhardwaj, A.; Kaur, J.; Sharma, S. K.; Huang, Z. J.; Wuest, F.; Knaus, E. E. Bioorg. Med. Chem. Lett. 2013, 23, 163. 